\title{
Algebraic curves and maximal arcs
}

\author{
A. Aguglia • L. Giuzzi • G. Korchmáros
}

Received: 26 February 2007 / Accepted: 15 January 2008 / Published online: 24 January 2008

(C) Springer Science+Business Media, LLC 2008

\begin{abstract}
A lower bound on the minimum degree of the plane algebraic curves containing every point in a large point-set $\mathcal{K}$ of the Desarguesian plane $\mathrm{PG}(2, q)$ is obtained. The case where $\mathcal{K}$ is a maximal $(k, n)$-arc is considered in greater depth.
\end{abstract}

Keywords Algebraic curves - Maximal arcs

\section{Introduction}

In finite geometry, plane algebraic curves of minimum degree containing a given large point-set $\mathcal{K}$ in $\operatorname{PG}(2, q)$ have been a useful tool to investigate combinatorial properties of $\mathcal{K}$.

When $\mathcal{K}$ is the whole point-set of $\operatorname{PG}(2, q)$, a trivial lower bound on the degree of such a plane algebraic curve is $q+1$. G. Tallini pointed out that this is attained only when the curve splits into $q+1$ distinct lines of $\operatorname{PG}(2, q)$, all passing through the same point. He also gave a complete classification of the absolutely irreducible curves of degree $q+2$ containing all points of $\mathrm{PG}(2, q)$; see [21, 22] and also [1]. If $\mathcal{K}$ is the complementary set of a line in $\operatorname{PG}(2, q)$, then the bound is $q$; see [11].

Research supported by the Italian Ministry MURST, Strutture geometriche, combinatoria e loro applicazioni.

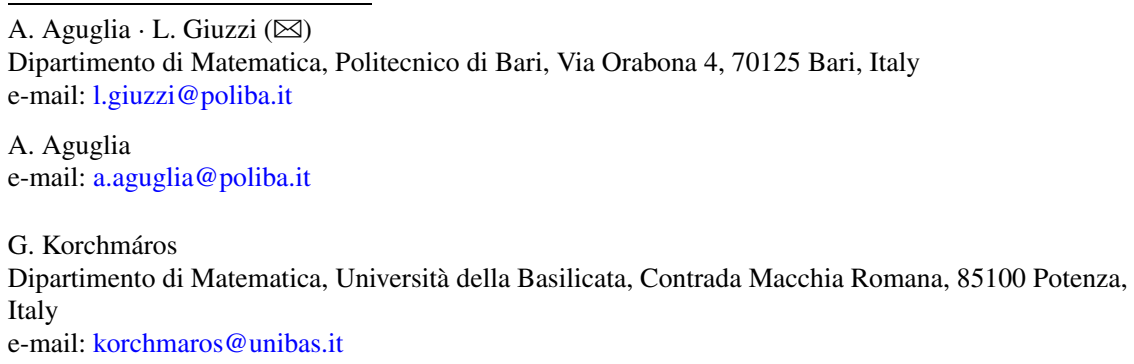


When $\mathcal{K}$ consists of all internal points to a conic $\mathcal{C}$ in $\operatorname{PG}(2, q)$ with $q$ odd, the above lower bound is $q-1$. The analogous bound for the set of the external points to $\mathcal{C}$ is $q$. These bounds were the main ingredients for recent combinatorial characterisations of point-sets blocking all external lines to $\mathcal{C}$; see $[4,10]$.

When $\mathcal{K}$ is a classical unital of $\mathrm{PG}(2, q)$, with $q$ square, the minimum degree $d$ of an absolutely irreducible curve $\mathcal{C}$ through $\mathcal{K}$ is $d=\sqrt{q}+1$. For non-classical unitals, the best known bound is $d>2 \sqrt{q}-4$; see [16].

Our purpose is to find similar bounds for slightly smaller, but still quite large, point-sets $\mathcal{K}$, say $|\mathcal{K}|=q t+\alpha$ with $t<c \sqrt[3]{q}$ and $0 \leq \alpha<q$, where $c$ is a suitable constant. Since no combinatorial condition on the configuration of $\mathcal{K}$ is assumed, we are relying on techniques and results from algebraic geometry rather than on the constructive methods used in the papers cited above.

The main result is that if $q>8 t^{3}-12^{2}+4 t-2 \alpha+2$, any plane algebraic curve $\Gamma$ containing every point of $\mathcal{K}$ has degree $d \geq 2 t$. The hypothesis on the magnitude of $t$ can be relaxed to $q>16 t^{2}-24 t-2 \alpha+8$ whenever $q$ is a prime.

In some cases, the bound $d \geq 2 t$ is sharp, as the following example shows. Let $\mathcal{K}$ be the union of $t$ disjoint ovals. If $q$ odd, or $q$ is even and the ovals are classical, then $|\mathcal{K}|=q t+t$ and $d=2 t$. The latter case is known to occur when $\mathcal{K}$ is a Denniston maximal arc [9] (or one of the maximal arcs constructed by Mathon and others [12, $14,18,23,24]$ ) minus the common nucleus of the ovals.

On the other hand, some refinement of the bound is also possible. Let $\mathcal{K}$ be any maximal arc of size $|\mathcal{K}|=q t+t+1$, that is, a $(q t+t+1, t+1)$-arc. Theorem 4.2 shows that if $q$ is large enough comparing to $t$, then no plane algebraic curve of degree $2 t$ passes through every point of $\mathcal{K}$. Therefore, the minimum degree is at least $2 t+1$ for such $t$ and this bound is attained when $\mathcal{K}$ is one of the above maximal arcs.

The case $n=4$ is considered in more detail. For $q>2^{6}$, the minimum degree is 7 and this is only attained when $\mathcal{K}$ is a Denniston arc and the curve $\Gamma$ of minimum degree splits into three distinct conics with the same nucleus $N \in \mathcal{K}$, together with a line through $N$.

\section{Some background on plane algebraic curves over a finite field}

A plane projective algebraic curve $\Delta$ over the algebraic closure $\overline{\mathrm{GF}(q)}$ of $\mathrm{GF}(q)$ is defined over $\operatorname{GF}(q)$ if it has an affine equation $f(X, Y)=0$ with $f(X, Y) \in$ $\operatorname{GF}(q)[X, Y]$. The curve $\Delta$ is absolutely irreducible if it is irreducible over the algebraic closure $\overline{\mathrm{GF}(q)}$. Denote by $N_{q}$ the number of non-singular points lying in $\mathrm{PG}(2, q)$ of an absolutely irreducible plane curve $\Delta$ of degree $d$. From the HasseWeil bound follows

$$
N_{q} \leq q+1+(d-1)(d-2) \sqrt{q} .
$$

This holds true when singular points of $\Delta$ lying in $\mathrm{PG}(2, q)$ are also counted; see [17].

The Stöhr-Voloch bound depends not only on the degree $d$, but also on a positive integer, the Frobenius order $v$ of $\Delta$; see [20]. This number $v$ is either 1 or $\varepsilon_{2}$, where $\varepsilon_{2}$ is the intersection number $I(P, \Delta \cap \ell)$ of $\Delta$ with the tangent line $\ell$ at a general point $P \in \Delta$. It turns out that $\varepsilon_{2}$ is either 2 , or a power, say $p^{h}$, of the characteristic 
$p$ of the plane, and is the minimum of $I(Q, \Delta \cap r)$, where $Q$ ranges over the nonsingular points of $\Delta$ and $r$ is the tangent to $\Delta$ at $Q$. If $q=p$, then $\varepsilon_{2}=2$, and either $v=1$, or $v=2$ and $p=2$. With this notation, the Stöhr-Voloch bound applied to $\Delta$ is

$$
2 N_{q} \leq v(d-3) d+d(q+2) .
$$

The following algebraic machinery can be used to compute $v$. Let $P=(a, b)$ be a non-singular point of $\Delta$ such that the tangent line to $\Delta$ at $P$ is not the vertical line through $P$. The unique branch (or place) centred at $P$ has a local parametrisation, also called a primitive branch representation,

$$
x=a+t, y=b+\varphi(t)
$$

where $f(x, y)=0$ and $\varphi(t)=b_{k} t^{k}+\ldots$ with $k \geq 1$ is a formal power series with coefficients in $\overline{\mathrm{GF}(q)}[[t]]$; see [19]. Then, $v$ is defined to be the smallest integer such that the determinant

$$
\left|\begin{array}{cc}
x-x^{q} & y-y^{q} \\
1 & D_{t}^{(v)}(y)
\end{array}\right|=\left|\begin{array}{cc}
a-a^{q}+t-t^{q} & b-b^{q}+\varphi(t)-\varphi(t)^{q} \\
1 & D_{t}^{(v)}(\varphi(t))
\end{array}\right|
$$

does not vanish. Here $D_{t}$ denotes the $\nu$-th Hasse derivative, that is,

$$
D_{t}^{(v)}(\varphi(t))=\left(\begin{array}{l}
k \\
v
\end{array}\right) b_{k} t^{k-v}+\ldots
$$

The above idea still works if osculating conics are used in place of tangent lines, and, in some cases, the resulting bound improves (2). Before stating the result, which is the Stöhr-Voloch bound for conics, a further concept from algebraic geometry is needed. Recall that the order sequence of $\Delta$ with respect to the linear system $\Sigma_{2}$ of the conics of the plane is the increasing sequence $0, \epsilon_{1}=1, \epsilon_{2}=2, \epsilon_{3}, \epsilon_{4}, \epsilon_{5}$ of all intersection numbers $I(P, \Delta \cap \mathcal{C})$ of $\Delta$ with conics at a general point $P$. The Frobenius $\Sigma_{2}$-order sequence is the subsequence $v_{0}=0, v_{1}, v_{2}, v_{3}, v_{4}$ extracted increasingly from the $\Sigma_{2}$-order sequence of $\Delta$, for which the following determinant does not vanish:

$$
\left|\begin{array}{ccccc}
x-x^{q} & x^{2}-x^{2 q} & y-y^{q} & x y-x^{q} y^{q} & y^{2}-y^{2 q} \\
1 & 2 x & D_{t}^{\left(\nu_{1}\right)}(y) & D_{t}^{\left(\nu_{1}\right)}(x y) & D_{t}^{\left(\nu_{1}\right)}\left(y^{2}\right) \\
0 & 1 & D_{t}^{\left(\nu_{2}\right)}(y) & D_{t}^{\left(\nu_{2}\right)}(x y) & D_{t}^{\left(\nu_{2}\right)}\left(y^{2}\right) \\
0 & 0 & D_{t}^{\left(\nu_{3}\right)}(y) & D_{t}^{\left(\nu_{3}\right)}(x y) & D_{t}^{\left(\nu_{3}\right)}\left(y^{2}\right) \\
0 & 0 & D_{t}^{\left(\nu_{4}\right)}(y) & D_{t}^{\left(\nu_{4}\right)}(x y) & D_{t}^{\left(\nu_{4}\right)}\left(y^{2}\right)
\end{array}\right| .
$$

Assume that deg $\Delta \geq 3$. The Stöhr-Voloch bound for conics, that is for $\Sigma_{2}$, is

$$
5 N_{q} \leq\left[\left(v_{1}+\ldots+v_{4}\right)(d-3) d+2 d(q+5)\right] .
$$

For more on the Stöhr-Voloch bound see [20]. 


\section{Plane algebraic curves of minimum degree through all the points of a given point-set}

In this section, $\mathcal{K}$ stands for a set of $q t+\alpha$ points in $\mathrm{PG}(2, q)$, with $0 \leq t \leq q$ and $0 \leq$ $\alpha<q$. Let $\Gamma$ denote a plane algebraic curve of degree $d$ containing every point $\mathcal{K}$. As already mentioned, $q+1$ is the minimum degree of a plane algebraic curve containing every point of $\operatorname{PG}(2, q)$. Thus, since we are looking for lower bounds on $d$, we will only be concerned with the case where $d \leq q$.

A straightforward counting argument gives the following result.

Lemma 3.1 If $d \leq q$, then $d \geq t$.

Proof Since $d \leq q$, the linear components of $\Gamma$ do not contain all the points of $\mathrm{PG}(2, q)$. Choose a point $P \in \mathrm{PG}(2, q)$ not in any of these linear components. Each of the $q+1$ lines through $P$ meets $\Gamma$ in at most $d$ distinct points. Thus, $(q+1) d \geq|\mathcal{K}|$, that is

$$
d \geq \frac{q t+\alpha}{q+1} \geq t-\frac{t}{q+1} .
$$

Since $t<q+1$, the assertion follows.

Our aim is to improve Lemma 3.1. Write $\mathfrak{F}$ for the set of all lines of $\operatorname{PG}(2, q)$ meeting $\mathcal{K}$ in at least 1 point. Set $m_{0}=\min \{|\ell \cap \mathcal{K}|: \ell \in \mathfrak{F}\}$ and $M_{0}=\max \{|\ell \cap \mathcal{K}|: \ell \in \mathfrak{F}\}$.

Theorem 3.2 Let $\Gamma$ be an algebraic plane curve over $\overline{\mathrm{GF}(q)}$ of minimal degree $d$ which passes through all the points of $\mathcal{K}$. If

$$
q>8 t^{3}-16 t^{2}+2 t+4-2 m_{0}\left(2 t^{2}-5 t+2\right)+2 M_{0}(2 t-1),
$$

then $\operatorname{deg} \Gamma \geq 2 t$. For prime $q$, Condition (4) may be relaxed to

$$
q>8 t^{2}-16 t+8-2 \alpha+2 M_{0}(2 t-1) .
$$

Proof We prove that if $d \leq 2 t-1$, then (4) does not hold. For $q$ prime we show that also (5) is not satisfied. Since $\Gamma$ is not necessarily irreducible, the following setup is required.

The curves $\Delta_{1}, \ldots, \Delta_{l}$ are the absolutely irreducible non-linear components of $\Gamma$ defined over $\operatorname{GF}(q)$, respectively of degree $d_{i} ; r_{1}, \ldots, r_{k}$ are the linear components of $\Gamma$ over $G F(q) ; \Xi_{1}, \ldots, \Xi_{s}$ are the components of $\Gamma$ which are irreducible over $\mathrm{GF}(q)$ but not over $\overline{\mathrm{GF}(q)}$.

The idea is to estimate the number of points in $\operatorname{PG}(2, q)$ that each of the above components can have.

Let $N_{i}$ be the number of non-singular points of $\Delta_{i}$ lying in $\operatorname{PG}(2, q)$. Then, (2) holds for any $\Delta_{i}$. Let $v^{(i)}$ denote the Frobenius order of $\Delta_{i}$. If $v=\max \left\{v^{(i)} \mid 1 \leq\right.$ $i \leq l\}$ and $\delta=\sum_{i=1}^{l} d_{i}$, then

$$
2 \sum_{i=1}^{l} N_{i} \leq \sum_{i=1}^{l} v^{(i)} d_{i}\left(d_{i}-3\right)+(q+2) d_{i} \leq v \delta(\delta-3)+(q+2) \delta .
$$


For $q$ prime, $v=1$; see [20]. Since $v^{(i)}=1$ can fail for $q>p$, an upper bound on $v^{(i)}$ depending on $d_{i}$ is needed. As $v^{(i)} \leq \varepsilon_{2}^{(i)}$, a bound on $\varepsilon_{2}^{(i)}$ suffices. Since $N_{i}>0$ may be assumed, $\Delta_{i}$ has a non-singular point $P$ lying in $\operatorname{PG}(2, q)$. If $\ell$ is the tangent to $\Delta_{i}$ at $P$, then

$$
d_{i}=\sum_{Q \in \ell \cap \Delta_{i}} I\left(Q, \ell \cap \Delta_{i}\right)=I\left(P, \ell \cap \Delta_{i}\right)+\sum_{\substack{Q \in \ell \cap \Delta_{i} \\ Q \neq P}} I\left(Q, \ell \cap \Delta_{i}\right) \geq \varepsilon_{2}^{(i)}+m_{0}-1
$$

whence $v^{(i)} \leq d_{i}-m_{0}+1$. From (6),

$$
2 \sum_{i=1}^{l} N_{i} \leq \begin{cases}\delta(\delta-3)+(q+2) \delta & q \geq 3 \text { prime } \\ \left(\delta-m_{0}+1\right) \delta(\delta-3)+(q+2) \delta & \text { otherwise }\end{cases}
$$

If $\Delta_{i}$ has $M_{i}$ singular points, from Plücker's theorem $M_{i} \leq \frac{1}{2}\left(d_{i}-1\right)\left(d_{i}-2\right)$. Hence,

$$
2 \sum_{i=1}^{l} M_{i} \leq \sum_{i=1}^{l}\left(d_{i}-1\right)\left(d_{i}-2\right) \leq(\delta-1)(\delta-2) .
$$

The number of points of $\mathcal{K}$ lying on linear components $r_{i}$ is at most $k M_{0} \leq d M_{0}$.

For every $\Xi=\Xi_{i}$, there exists an absolutely irreducible curve $\Theta$, defined over the algebraic extension $\mathrm{GF}\left(q^{\xi}\right)$ of degree $\xi>1$ of $\mathrm{GF}(q)$ in $\overline{\mathrm{GF}(q)}$, such that the absolutely irreducible components of $\Xi$ are $\Theta$ and its conjugates $\Theta_{1}, \ldots, \Theta_{\xi-1}$. Here, if $\Theta$ has equation $\sum a_{k j} X^{k} Y^{j}=0$ and $1 \leq w \leq \xi-1$, then $\Theta_{w}$ is the curve of equation $\sum a_{k j}^{q^{w}} X^{k} Y^{j}=0$. Since $\Xi, \Theta$ and the conjugates of $\Theta$ pass through the same points in PG(2,q), from Bézout's theorem, see [15, Lemma 2.24], $\Xi$ has at most $\theta^{2}$ points in $\operatorname{PG}(2, q)$ where $\theta=\operatorname{deg} \Theta$. Note that $\operatorname{deg} \Xi \geq 2 \theta$.

Let $N_{i}^{\prime}$ denote the total number of points (simple or singular) of $\Xi_{i}$ lying in $\mathrm{PG}(2, q)$. From the above argument,

$$
\sum_{i=1}^{s} N_{i}^{\prime} \leq \sum_{i=1}^{s} \theta_{i}^{2}<\left(\sum_{i=1}^{s} \theta_{i}\right)^{2} \leq \frac{1}{4}\left(\sum_{i=1}^{s} \operatorname{deg} \Xi_{i}\right)^{2}<\frac{1}{2}(q+2) \sum_{i=1}^{s} \operatorname{deg} \Xi_{i} .
$$

As

$$
q t+\alpha \leq \sum_{i=1}^{l}\left(N_{i}+M_{i}\right)+k M_{0}+\sum_{i=1}^{s} N_{i}^{\prime},
$$

from (7), (8), (9) and (10) it follows that

$$
2(q t+\alpha) \leq \begin{cases}2 d^{2}-6 d+2+2 d M_{0}+(q+2) d & q \geq 3 \text { prime } \\ d^{3}-d^{2}-6 d+2-m_{0} d(d-3)+2 d M_{0}+(q+2) d & \text { otherwise }\end{cases}
$$

Since $d \leq 2 t-1$, the main assertion follows by straightforward computation. 
Remark 3.3 As $1 \leq m_{0} \leq M_{0} \leq d$, the proof of Theorem 3.2 shows that Condition (4), and, for $q$ prime, Condition (5), may be replaced by the somewhat weaker, but more manageable, condition $q>8 t^{3}-12^{2}+4 t-2 \alpha+2\left(\right.$ and $q>16 t^{2}-24 t-2 \alpha+8$ for $q$ prime).

Remark 3.4 As pointed out in the Introduction, Theorem 3.2 is sharp as the bound is attained by some maximal $(k, n)$-arcs.

Corollary 3.5 If $\Gamma$ has a component not defined over $\mathrm{GF}(q)$, then

$$
\operatorname{deg} \Gamma \geq 2 t+1
$$

Proof We use the same arguments as in the proof of Theorem 3.2, considering that $\delta<d$. In particular, we have

$$
2(q t+\alpha)<d^{3}-\left(4+m_{0}\right) d^{2}+\left(q+5 m_{0}+1\right) d+\left(4-4 m_{0}-q\right)+2 d M_{0},
$$

which for $d \leq 2 t$ proves the assertion.

Remark 3.6 Corollary 3.5 implies that a plane algebraic curve of degree $2 t$ containing $\mathcal{K}$ is always defined over $\mathrm{GF}(q)$.

Theorem 3.7 If the curve $\Gamma$ in Theorem 3.2 has no quadratic component, and

$$
q>\frac{750 t^{3}-1725 t^{2}+10\left(10 M_{0}+113\right) t-184-40\left(\alpha+M_{0}\right)}{40},
$$

then

$$
d \geq \frac{5}{2} t
$$

If $q>5$ is prime, then Condition (11) may be relaxed to

$$
q>\frac{125 t^{2}+2\left(10 M_{0}-105\right) t-8\left(\alpha+M_{0}-9\right)}{8}
$$

Proof We prove that if $d \leq \frac{5}{2}(t-1)$, then (11) (and, for $q$ prime, (12)) does not hold. It is sufficient to just change slightly the proof of Theorem 3.2. Let $v_{0}^{(i)}=0$, $\ldots, v_{4}^{(i)}$ be the Frobenius orders of $\Delta_{i}$ with respect to conics. If $q$ is a prime greater than 5 , then $v_{j}^{(i)}=j$ for $0 \leq j \leq 4$. Otherwise, set $v^{(i)}=\sum_{j=1}^{4} v_{j}^{(i)}$. Since $v^{(i)} \leq$ $2+\varepsilon_{3}+\varepsilon_{4}+\varepsilon_{5}$ and $\varepsilon_{5} \leq 2 d_{i}$, we have that $\nu^{(i)} \leq 6 d_{i}-1$. From (3),

$$
5 \sum_{i=1}^{l} N_{i} \leq \begin{cases}10 \delta(\delta-3)+(q+5) 2 \delta & q>5 \text { prime } \\ (6 \delta-1) \delta(\delta-3)+(q+5) 2 \delta, & \text { otherwise. }\end{cases}
$$


Using the same argument as in (10),

$$
\sum_{i=1}^{s} N_{i}^{\prime} \leq \frac{1}{4}\left(\sum_{i=1}^{s} \operatorname{deg} \Xi_{i}\right)^{2}<\frac{2}{5}(q+5) \sum_{i=1}^{s} \operatorname{deg} \Xi_{i} .
$$

Using now (8), (9), (13) and (14) we obtain

$$
(q t+\alpha) \leq \begin{cases}\frac{5}{2} d^{2}-\frac{11}{2} d+d M_{0}+\frac{2}{5} q d+1 & q>5 \text { prime } \\ \frac{6}{5} d^{3}-\frac{33}{10} d^{2}+\frac{11}{10} d+d M_{0}+\frac{2}{5} q d+1 & \text { otherwise }\end{cases}
$$

Then, (15) does not hold for any $q$. If $q$ is prime, also (12) is not satisfied.

\section{Algebraic curves passing through the points of a maximal arc}

Remark 3.4 motivates the study of plane algebraic curves passing through all the points of maximal $(k, n)$-arc in $\operatorname{PG}(2, q)$.

In this section $\mathcal{K}$ always denotes a maximal $(k, n)$-arc. Recall that a $(k, n)$-arc $\mathcal{K}$ of a projective plane $\pi$ is a set of $k$ points, no $n+1$ collinear. Barlotti [8] proved that $k \leq(n-1) q+n$, for any $(k, n)$-arc in $\mathrm{PG}(2, q)$; when equality holds, a $(k, n)$ arc is maximal. A purely combinatorial property characterising a $(k, n)$-maximal arc $\mathcal{K}$ is that every line of $\operatorname{PG}(2, q)$ either meets $\mathcal{K}$ in $n$ points or is disjoint from it. Trivial examples of maximal arcs in $\mathrm{PG}(2, q)$ are the $\left(q^{2}+q+1, q+1\right)$-arc given by all the points of $\operatorname{PG}(2, q)$ and the $\left(q^{2}, q\right)$-arcs consisting of the points of an affine subplane $\mathrm{AG}(2, q)$ of $\mathrm{PG}(2, q)$. Ball, Blokhuis and Mazzocca [6, 7] have shown that no non-trivial maximal arc exists in $\mathrm{PG}(2, q)$ for $q$ odd. On the other hand, for $q$ even, several maximal arcs exist in the Desarguesian plane and many constructions are known; see [9, 12, 14, 18, 23, 24]. The arcs arising from these constructions, with the exception of those of [23], see also [14], all consist of the union of $n-1$ disjoint conics together with their common nucleus $N$. In other words, these arcs are covered by a completely reducible curve of degree $2 n-1$, whose components are $n-1$ conics and a line through the point $N$.

Remark 4.1 From Corollary 3.5, if $\Gamma$ has a component defined over $\overline{\mathrm{GF}(q)}$ but not over $\operatorname{GF}(q)$, and it passes through all the points of $\mathcal{K}$, then its degree $d$ is at least $2 n-1$.

The following theorem shows that the above hypothesis on the components of $\Gamma$ can be dropped as far as $q$ is sufficiently large.

Theorem 4.2 For any $n$, there exists $q_{0} \leq(2 n-2)^{2}$ such that any plane algebraic curve $\Gamma$ defined over $\operatorname{GF}(q)$ with $q>q_{0}$, passing through all the points of a maximal $(k, n)$-arc $\mathcal{K}$ of $\operatorname{PG}(2, q)$ has degree $d \geq 2 n-1$. If equality holds, then $\Gamma$ has either one linear and $n-1$ absolutely irreducible quadratic components or $n-2$ absolutely irreducible quadratic components and one cubic component. 
The proof depends on the following lemma.

Lemma 4.3 Assume that $\Gamma$ is reducible and that the number of its components is less than $n-1$. Then, the degree $d$ of $\Gamma$ satisfies

$$
d \geq \sqrt[4]{q}
$$

Proof We use the same setup as in the proof of Theorem 3.2. This time the HasseWeil bound is used in place of the Stöhr-Voloch bound. The number of points of $\Delta_{i}$ in $\mathrm{PG}(2, q)$ is $N_{i}+M_{i}$; from (1),

$$
M_{i}+N_{i} \leq q+1+\left(d_{i}-1\right)\left(d_{i}-2\right) \sqrt{q} .
$$

From (9),

$$
\sum_{i=1}^{s} N_{i}^{\prime}<(q+1)+\sum_{i=1}^{s}\left(\operatorname{deg} \Xi_{i}-1\right)\left(\operatorname{deg} \Xi_{i}-2\right) \sqrt{q} .
$$

If $\Gamma$ has $w$ components, then

$$
w(q+1)+\sum_{i=1}^{s}\left(\operatorname{deg} \Xi_{i}-1\right)\left(\operatorname{deg} \Xi_{i}-2\right) \sqrt{q}+\sum_{j=1}^{l}\left(d_{i}-1\right)\left(d_{i}-2\right) \sqrt{q} \geq(n-1) q+n .
$$

Since $w \leq n-2$, (18) yields

$$
(d-1)(d-2) \sqrt{q} \geq q+2
$$

hence, $d \geq \sqrt[4]{q}$.

Proof of Theorem 4.2 Suppose $\Gamma$ to have degree $d<2 n-1$. By Remark 4.1, all components of $\Gamma$ are defined over $\operatorname{GF}(q)$. If $\Gamma$ is absolutely irreducible, then (1) implies that $\Gamma$ contains at most $q+(2 n-3)(2 n-4) \sqrt{q}+1$ points. However, for $q$ large enough, this number is less than $(n-1) q+n$; a contradiction.

When $\Gamma$ has more then one component, denote by $t_{j}$ the number of its components of degree $j$. Let $u$ be the maximum degree of such components. Then, $u \leq 2 n-3$ and

$$
d=\sum_{j=1}^{u} j t_{j} \leq 2 n-2
$$

From (1),

$$
|\Gamma \cap \mathcal{K}| \leq n t_{1}+\sum_{j=2}^{u} t_{j}(q+(j-1)(j-2) \sqrt{q}+1)=\left(\sum_{j=2}^{u} t_{j}\right) q+c \sqrt{q}+d,
$$

where

$$
c=\sum_{j=2}^{u} t_{j}(j-1)(j-2), \quad d=n t_{1}+\sum_{j=2}^{u} t_{j}
$$


Both $c$ and $d$ are independent from $q$; therefore,

$$
n-1=\lim _{q \rightarrow \infty} \frac{|\Gamma \cap \mathcal{K}|}{q}=\sum_{j=2}^{u} t_{j}
$$

Hence,

$$
2(n-1)=2 \sum_{j=2}^{u} t_{j} \leq t_{1}+\sum_{j=2}^{u} j t_{j}=d .
$$

Since $d \leq 2 n-2$, by Lemma 4.3 , for $q>(2 n-2)^{4}$ the curve $\Gamma$ should have at least $n-1$ components. This would imply that either $u=2, t_{1}=1, t_{2}=(n-1)$ or $u=3$, $t_{1}=0, t_{2}=(n-2), t_{3}=1$. In particular, in both cases $d=2 n-1$.

Remark 4.4 As mentioned in the Introduction, case $d=2 n-1$ in Theorem 4.2 occurs when $\mathcal{K}$ is a Denniston maximal arc [9] (or one of the maximal arcs constructed by Mathon and others [12, 14, 18, 23, 24]). This result may not extend to any of the other known maximal arcs; they are the Thas maximal $\left(q^{3}-q^{2}+q, q\right)$-arcs in $\mathrm{PG}\left(2, q^{2}\right)$ arising from the Suzuki-Tits ovoid of $\mathrm{PG}(3, q)$; see [13, 23]. In fact, 22 is the minimum degree of a plane curve which passes through all points of a Thas' maximal $(456,8)$-arc in $\mathrm{PG}(2,64)$; see [3].

\section{Maximal arcs of degree 4}

From Theorem 4.2, for $q>6^{4}$, a lower bound on the degree of an algebraic curve $\Gamma$ passing through all the points of a maximal arc $\mathcal{K}$ of degree 4 is 7 . Our aim is to prove in this case the following result.

Theorem 5.1 Let $\mathcal{K}$ be a maximal arc of degree 4 and suppose there exists an algebraic plane curve $\Gamma$ containing all the points of $\mathcal{K}$. If $\operatorname{deg} \Gamma=7$, then $\Gamma$ consists of three disjoint conics, all with the same nucleus $N$, and a line through $N$.

Proof From Theorem 4.2, the curve $\Gamma$ splits either into one irreducible cubic and two irreducible conics or into three irreducible conics and one line $r$. These two cases are investigated separately.

Let $C$ denote any of the above conics of nucleus $N$. We show that every point of $C^{\prime}=C \cup\{N\}$ lying in $\mathrm{PG}(2, q)$ is contained in $\mathcal{K}$ : in fact, if there were a point $P \in C^{\prime} \backslash \mathcal{K}$, then there would be at least $\frac{q}{4}$ lines through $P$ external to $\mathcal{K}$. All these lines would meet $C^{\prime}$ in $\frac{q}{4}$ distinct points, which, in turn, would not be on $\mathcal{K}$. Hence, $\Gamma$ would have less than $3 q+4$ points on the arc $\mathcal{K}$, a contradiction.

Now assume that $\Gamma$ splits into a cubic $\mathcal{D}$ and two conics $C_{i}$, with $i=1,2$. Denote by $N_{i}$ the nucleus of $C_{i}$ and set $\mathcal{X}=C_{1} \cup C_{2} \cup\left\{N_{1}, N_{2}\right\}$. Since $|\mathcal{X}| \leq 2 q+4$, there exists a point $P \in \mathcal{K} \backslash \mathcal{X}$. Obviously, $P \in \mathcal{D}$. Every line through $P$ meets $\mathcal{K}$ in four points; thus, there is no line $\ell$ through $P$ meeting both $C_{1}$ and $C_{2}$ in 2 points; otherwise, $|\ell \cap \mathcal{X} \cap \mathcal{K}|=4$ and $|\ell \cap \mathcal{K}| \geq 5$, a contradiction. Hence, there are at least $q-1$ 
Fig. 1 Case 1 in Theorem 5.1

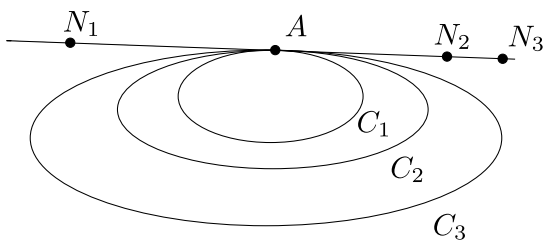

Fig. 2 Case 2 in Theorem 5.1

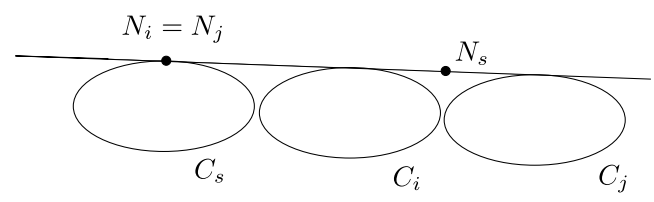

Fig. 3 Case 3 in Theorem 5.1

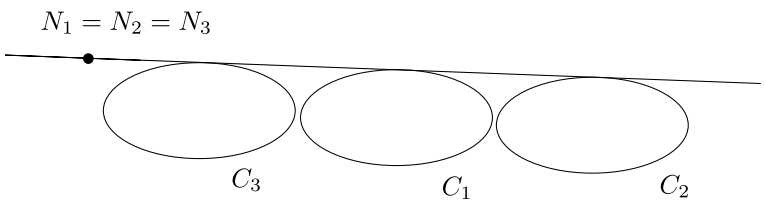

lines through $P$ meeting $\mathcal{D}$ in another point $P^{\prime}$. There are at most 5 bisecants to the irreducible cubic curve $\mathcal{D}$ through any given point $P \in \mathcal{D}$, namely the tangent in $P$ to $\mathcal{D}$ and, possibly, four other tangents in different points to $\mathcal{D}$ passing through $P$. Hence, there are $q-6$ lines through $P$ meeting $\mathcal{D}$ in three points. If this were the case, $\mathcal{D}$ would consist of at least $2(q-6)+6$ points, which is impossible. Therefore, we may assume that $\Gamma$ splits into three conics, say $C_{1}, C_{2}, C_{3}$, with nuclei $N_{1}, N_{2}$, $N_{3}$, and a line $r$.

Recall that, as seen above, the nuclei of all the conics belong to $\mathcal{K}$. Now we show that at least one nucleus, say $N_{1}$, lies on the line $r$. Since $\Gamma$ is a curve containing $\mathcal{K}$ of minimum degree with respect to this property, there is at least a point $P$ on $r \cap \mathcal{K}$ not on $C_{1}$. Each line through $P$ is a 4-secant to $\mathcal{K}$ hence, it meets $\mathcal{X}$ in an odd number of points. If $P \neq N_{1}$, then the number of lines through $P$ meeting $\mathcal{X}$ in an odd number of points is at most 15 , which is less than $q+1$ for $q \geq 2^{4}$, a contradiction. Actually, all the nuclei $N_{i}$ lie on $r$. In fact, suppose that $N_{j} \notin r$ for $j \in\{2,3\}$. Then, $N_{j} \in C_{s}$, with $s \neq 1, j$ and the line $N_{1} N_{j}$ joining $N_{1}$ and $N_{j}$ is tangent to $C_{1}$ and $C_{j}$. Consequently, $N_{1} N_{j}$ meets $C_{s}$ in another point different from $N_{j}$ that is, it is a 5-secant to $\mathcal{K}$, again a contradiction.

We are left with three cases, namely:

1. $N_{1} \neq N_{2} \neq N_{3}, C_{i} \cap C_{j}=\{A\}$ for any $i \neq j$ and $A \in N_{1} N_{2}$.

2. $N_{i}=N_{j}, N_{j} \neq N_{s}, N_{i} \in C_{s}, C_{i} \cap C_{j}=\emptyset$, with $i, j, s \in\{1,2,3\}$.

3. $N_{1}=N_{2}=N_{3}$ and $C_{i} \cap C_{j}=\emptyset$ for $i \neq j$.

We are going to show that cases (1) and (2) do not actually occur.

Lemma 5.2 Let $C_{1}, C_{2}$ be two conics with a common point $A$ but different nuclei $N_{1}, N_{2}$. If $A \in N_{1} N_{2}$ then there is a line $s$ with $A \in s$ and $s \neq N_{1} N_{2}$ such that for 
any point on $s \backslash\left(C_{1} \cup C_{2}\right)$ there passes a line $\ell$ with

$$
\left|\ell \cap\left(C_{1} \cup C_{2} \cup\left\{N_{1}, N_{2}\right\}\right)\right| \geq 3 .
$$

Proof Let $(X, Y, Z)$ denote homogeneous coordinates of points of the plane $\mathrm{PG}(2, q)$. Choose a reference system such that $A=O=(0,0,1)$ and the line joining $N_{1}$ and $N_{2}$ is the $X$-axis. We may suppose $C_{i}$ to have equation

$$
\alpha_{i} X^{2}+X Y+\beta_{i} Y^{2}+\lambda_{i} Y Z=0,
$$

where $\alpha_{i}, \beta_{i}, \lambda_{i} \in \mathrm{GF}(q)$ and $i=1,2$.

Since both $C_{i}$ are non-degenerate conics, we have $\alpha_{i} \neq 0$ and $\lambda_{i} \neq 0$. Furthermore, $\lambda_{1} \neq \lambda_{2}$ as the nuclei $N_{1}$ and $N_{2}$ are distinct.

Denote by $\mathfrak{T}(x)$ the trace of $\operatorname{GF}\left(q^{2}\right)$ over $\operatorname{GF}(2)$. If $\mathfrak{T}\left(\left(\alpha_{1} \lambda_{2}+\alpha_{2} \lambda_{1}\right)\left(\beta_{1} \lambda_{2}+\right.\right.$ $\left.\left.\beta_{2} \lambda_{1}\right)\right)=0$, then the two conics $C_{1}$ and $C_{2}$ have more than one point in common, which is impossible. Hence, $\left.\mathfrak{T}\left(\alpha_{1} \lambda_{2}+\alpha_{2} \lambda_{1}\right)\left(\beta_{1} \lambda_{2}+\beta_{2} \lambda_{1}\right)\right)$ $=1$; in particular, $\beta_{1} \lambda_{2} \neq \beta_{2} \lambda_{1}$.

A generic point $P_{m}^{i}$ of $C_{i} \backslash\{A\}$ has homogeneous coordinates

$$
P_{m}^{i}=\left(\frac{\lambda_{i} m}{\alpha_{i} m^{2}+m+\beta_{i}}, \frac{\lambda_{i}}{\alpha_{i} m^{2}+m+\beta_{i}}, 1\right),
$$

with $m \in \mathrm{GF}(q) \backslash\{0\}$. Consider now a point $P_{\varepsilon}=(0, \epsilon, 1)$ on the $Y$-axis, with $\varepsilon \in$ $\mathrm{GF}(q) \backslash\left\{0, \frac{\lambda_{1}}{\beta_{1}}, \frac{\lambda_{2}}{\beta_{2}}\right\}$. The points $P_{m}^{1}, P_{t}^{2}$ and $P_{\varepsilon}$ are collinear if and only if

$$
\left|\begin{array}{ccc}
\frac{\lambda_{1} m}{\alpha_{1} m^{2}+m+\beta_{1}} & \frac{\lambda_{1}}{\lambda_{2} t} & 1 \\
\frac{\lambda_{1} m^{2}+m+\beta_{1}}{\alpha_{2} t^{2}+t+\beta_{2}} & \frac{\lambda_{2}}{\alpha_{2} t^{2}+t+\beta_{2}} & 1 \\
0 & \varepsilon & 1
\end{array}\right|=0,
$$

that is

$\alpha_{1} \lambda_{2} \varepsilon m^{2} t+\alpha_{2} \lambda_{1} \epsilon m t^{2}+\varepsilon\left(\lambda_{2}+\lambda_{1}\right) m t+\left(\varepsilon \lambda_{1} \beta_{2}+\lambda_{1} \lambda_{2}\right) m+\left(\epsilon \lambda_{2} \beta_{1}+\lambda_{1} \lambda_{2}\right) t=0$.

Equation (20) may be regarded as the affine equation of a cubic curve $\mathcal{D}$ in the indeterminate $m$ and $t$. Observe that $(0,0,1) \in \mathcal{D}$. The only points at infinity of $\mathcal{D}$ are $Y_{\infty}=(0,1,0), X_{\infty}=(0,1,0)$ and $B=\left(\frac{\lambda_{1} \alpha_{2}}{\lambda_{2} \alpha_{1}}, 1,0\right)$. Therefore, $\mathcal{D}$ does not split into three conjugate complex lines. Thus, by [15, Theorem 11.34] and [15, Theorem 11.46], there is at least one affine point $T=(\bar{m}, \bar{t}, 1)$ on $\mathcal{D}$ different from $(0,0,1)$.

Since $\mathfrak{T}\left(\left(\alpha_{1} \lambda_{2}+\alpha_{2} \lambda_{1}\right)\left(\beta_{1} \lambda_{2}+\beta_{2} \lambda_{1}\right)\right)=1$, the line $m=t$ is a 1 -secant to $\mathcal{D}$ in $(0,0,1)$; hence, the point $T$ is not on this line.

This implies that, for any given $\varepsilon \in \mathrm{GF}(q) \backslash\{0\}$, there exist at least two distinct values $\bar{m}, \bar{t} \in \mathrm{GF}(q) \backslash\{0\}$ satisfying (20). Hence, $P_{\bar{m}}, P_{\bar{t}}^{2}$ and $P_{\varepsilon}$ are collinear and the line $P_{\bar{m}}^{1} P_{\bar{t}}^{2}$ meets $C_{1} \cup C_{2} \cup\left\{N_{1}, N_{2}\right\}$ in at least three points.

From Lemma 5.2, in case (1) the set $C_{1} \cup C_{2} \cup\left\{N_{1}, N_{2}\right\}$ cannot be completed to a maximal arc just by adding a third conic $\mathcal{C}_{3}$, together with its nucleus $N_{3}$, since, in 
this case, there would be at least a 5-secant to $C_{1} \cup C_{2} \cup C_{3} \cup\left\{N_{1}, N_{2}, N_{3}\right\}$. Hence, case (1) is ruled out.

Lemma 5.3 Given any two disjoint conics $C_{1}, C_{2}$ with the same nucleus $N$, there is a unique degree-4 maximal arc containing $\mathcal{X}=C_{1} \cup C_{2}$.

Proof There is a line $r$ in $\mathrm{PG}(2, q)$ external to $\mathcal{X}$, since, otherwise, $\mathcal{X}$ would be a 2-blocking set with less than $2 q+\sqrt{2 q}+1$ points, which is a contradiction; see [5].

Choose a reference system such that $N=O=(0,0,1)$ and $r$ is the line at infinity $Z=0$. The conics $C_{i}$, for $i=1,2$, have equation:

$$
\alpha_{i} X^{2}+X Y+\beta_{i} Y^{2}+\lambda_{i} Z^{2}=0,
$$

where $\alpha_{i}, \beta_{i}, \lambda_{i} \in \mathrm{GF}(q)$ and $\mathfrak{T}\left(\alpha_{i} \beta_{i}\right)=1$.

Since both $C_{i}$ are non-degenerate, $\lambda_{i} \neq 0$. We first show that, $\lambda_{1} \neq \lambda_{2}$, as $C_{1}$ and $C_{2}$ are disjoint. We argue by contradiction. If $\lambda_{1}=\lambda_{2}$, then we could assume $\alpha_{1} \neq \alpha_{2}$; in fact, if $\alpha_{1}=\alpha_{2}$ and $\lambda_{1}=\lambda_{2}$, the linear system generated by $C_{1}$ and $C_{2}$ would contain the line $Y=0$; thus their intersection would not be empty. Let now

$$
\gamma=\sqrt{\frac{\beta_{1}-\beta_{2}}{\alpha_{1}-\alpha_{2}}} ;
$$

hence,

$$
\alpha_{1} \gamma^{2}+\gamma+\beta_{1}=\alpha_{2} \gamma^{2}+\gamma+\beta_{2},
$$

and the line $X=\gamma Y$ would meet the two conics in the same point

$$
P=\left(\gamma\left(\frac{\lambda_{1}}{\alpha_{1} \gamma^{2}+\gamma+\beta_{1}}\right)^{\frac{1}{2}},\left(\frac{\lambda_{1}}{\alpha_{1} \gamma^{2}+\gamma+\beta_{1}}\right)^{\frac{1}{2}}, 1\right),
$$

contradicting $C_{1} \cap C_{2}=\emptyset$.

We also see that $\alpha_{1} \lambda_{2} \neq \alpha_{2} \lambda_{1}$ and $\beta_{1} \lambda_{2} \neq \beta_{2} \lambda_{1}$, since, otherwise, the points

$$
P=\left(\sqrt{\frac{\lambda_{1}}{\alpha_{1}}}, 0,1\right)=\left(\sqrt{\frac{\lambda_{2}}{\alpha_{2}}}, 0,1\right), \quad Q=\left(0, \sqrt{\frac{\lambda_{1}}{\beta_{1}}}, 1\right)=\left(0, \sqrt{\frac{\lambda_{2}}{\beta_{2}}}, 1\right)
$$

would lie on both conics.

Let now $C_{3}$ be the conic with equation

$$
\frac{\alpha_{1} \lambda_{2}+\alpha_{2} \lambda_{1}}{\lambda_{1}+\lambda_{2}} X^{2}+X Y+\frac{\beta_{1} \lambda_{2}+\beta_{2} \lambda_{1}}{\lambda_{1}+\lambda_{2}} Y^{2}+\left(\lambda_{1}+\lambda_{2}\right) Z^{2}=0 .
$$

Set

$$
v=\frac{\left(\alpha_{1} \lambda_{2}+\alpha_{2} \lambda_{1}\right)\left(\beta_{1} \lambda_{2}+\beta_{2} \lambda_{1}\right)}{\lambda_{1}^{2}+\lambda_{2}^{2}} .
$$

The collineation $\mathrm{H}$ of $\mathrm{PG}(2, q)$ given by the matrix

$$
\left(\begin{array}{ccc}
a^{-1} & 0 & 0 \\
0 & a & 0 \\
b & c & 1
\end{array}\right)
$$


where $a=\sqrt{\frac{\alpha_{1} \lambda_{2}+\alpha_{2} \lambda_{1}}{\lambda_{1}+\lambda_{2}}}, b=\sqrt{\frac{1+\alpha_{1} / a^{2}}{\lambda_{1}}}$ and $c=a \sqrt{\frac{\beta_{1}+\beta_{2}}{\lambda_{1}+\lambda_{2}}}$, maps the conics $C_{i}, i=$ 1,2 , to

$$
\bar{C}_{2}^{i}: X^{2}+X Y+v Y^{2}+\lambda_{i} Z^{2}=0
$$

and $C_{3}$ to

$$
\bar{C}_{2}^{3}: X^{2}+X Y+v Y^{2}+\left(\lambda_{1}+\lambda_{2}\right) Z^{2}=0 .
$$

If $\mathfrak{T}(\nu)=0$, then $\bar{C}_{2}^{1}$ and $\bar{C}_{2}^{2}$ would share some point in common on the line at infinity. Hence, $\mathfrak{T}(v)=1$. In particular, $\bar{C}_{2}^{1}, \bar{C}_{2}^{2}$ and $\bar{C}_{2}^{3}$ together with their common nucleus $O$, form a degree-4 maximal arc $\mathcal{K}$ of Denniston type; see [2,9] and [18, Theorem 2.5].

It remains to show the uniqueness of $\mathcal{K}$. We first observe that $\mathcal{X}$ is a $(0,2,4)$-set with respect to lines of the plane. No point lying on a 4 -secant to $\mathcal{X}$ can be added to $\mathcal{X}$ to get a degree-4 maximal arc.

Take $P \notin \mathcal{X}$ and denote by $u_{i}$ with $i=0,2,4$, the number of $i$-secants to $\mathcal{X}$ through $P$, that is the number of lines meeting $\mathcal{X}$ in $i$ points. The lines through $P$ which are external to $\mathcal{X}$ are also external to $\mathcal{K}$ and the converse also holds. Therefore, when $P \notin \mathcal{K}$, we have $u_{0}=\frac{1}{4} q$. From

$$
\begin{aligned}
& u_{0}+u_{2}+u_{4}=q+1, \\
& 2 u_{2}+4 u_{4}=2 q+2,
\end{aligned}
$$

also $u_{4}=\frac{1}{4} q$. Hence, no point $P \notin \mathcal{K}$ may be added to $\mathcal{X}$ to obtain a maximal arc of degree 4 .

Finally, Lemma 5.3 shows that case (2) does not occur.

\section{References}

1. Abatangelo, V., Korchmáros, G.: A generalization of a theorem of B. Segre on regular points with respect to an ellipse of an affine Galois plane. Ann. Mat. Pure Appl. 72, 87-102 (1997)

2. Abatangelo, V., Larato, B.: A characterization of Denniston's maximal arcs. Geom. Dedicata 30, 197203 (1989)

3. Aguglia, A., Giuzzi, L.: An algorithm for constructing some maximal arcs in $\mathrm{PG}\left(2, q^{2}\right)$. Results Math., to appear. http://arxiv.org/abs/math/0611466

4. Aguglia, A., Korchmáros, G.: Blocking sets of external lines to a conic in $\mathrm{PG}(2, q), q$ odd. Combinatorica 26(4), 379-394 (2006)

5. Ball, S., Blokhuis, A.: On the size of a double blocking set in $\operatorname{PG}(2, q)$. Finite Fields Appl. 2(2), 125-137 (1996)

6. Ball, S., Blokhuis, A.: An easier proof of the maximal arcs conjecture. Proc. Am. Math. Soc. 126(11), 3377-3380 (1998)

7. Ball, S., Blokhuis, A., Mazzocca, F.: Maximal arcs in Desarguesian planes of odd order do not exist. Combinatorica 17(1), 31-41 (1997)

8. Barlotti, A.: Sui $(k, n)$-archi di un piano lineare finito. Boll. Un. Mat. Ital. 11(3), 553-556 (1956)

9. Denniston, R.H.F.: Some maximal arcs in finite projective planes. J. Comb. Theory 6, 317-319 (1969)

10. Giulietti, M.: Blocking sets of external lines to a conic in $\mathrm{PG}(2, q), q$ even. Eur. J. Comb. 28(1), 36-42 (2007)

11. Goppa, V.D.: Geometry and Codes. Kluwer, Amsterdam (1988) 
12. Hamilton, N., Mathon, R.: More maximal arcs in Desarguesian projective planes and their geometric structure. Adv. Geom. 3, 251-261 (2003)

13. Hamilton, N., Penttila, T.: A Characterisation of Thas maximal arcs in translation planes of square order. J. Geom. 51(1-2), 60-66 (1994)

14. Hamilton, N., Penttila, T.: Groups of maximal arcs. J. Comb. Theory Ser. A 94(1), 63-86 (2001)

15. Hirschfeld, J.W.P.: Projective Geometries over Finite Fields, 2nd edn. OUP, Oxford (1998)

16. Hirschfeld, J.W.P., Korchmáros, G.: Arcs and curves over finite fields. Finite Fields Appl. 5(4), 393408 (1999)

17. Leep, D.B., Yeomans, C.C.: The number of points on a singular curve over a finite field. Arch. Math. (Basel) 63(5), 420-426 (1994)

18. Mathon, R.: New maximal arcs in Desarguesian planes. J. Comb. Theory Ser. A 97(2), 353-368 (2002)

19. Seidenberg, A.: Elements of the Theory of Algebraic Curves. Addison-Wesley, Reading (1968)

20. Stöhr, K.O., Voloch, J.F.: Weierstrass points and curves over finite fields. Proc. Lond. Math. Soc. (3) 52(1), 1-19 (1986)

21. Tallini, G.: Sulle ipersuperficie irriducibili d'ordine minimo che contengono tutti i punti di uno spazio di Galois $S_{r, q}$. Rend. Mat. Appl. (5) 20, 431-479 (1961)

22. Tallini, G.: Le ipersuperficie irriducibili d'ordine minimo che invadono uno spazio di Galois. Atti Accad. Naz. Lincei Rend. Cl. Sci. Fis. Mat. Nat. (8) 30, 706-712 (1961)

23. Thas, J.A.: Construction of maximal arcs and partial geometries. Geom. Dedicata 3, 61-64 (1974)

24. Thas, J.A.: Construction of maximal arcs and dual ovals in translation planes. Eur. J. Comb. 1(2), 189-192 (1980) 